\title{
Uber Humussäuren;
}

von

\section{A. J van Schermbeck.}

Im Bande 70 dieser Zeitschrift brachten die Herren $\mathbf{P h}$. Malkomesius und R. Albert einige vorläufige Mitteilungen über Humussäuren. - Der Raum dieser Zeitschrift gestattet nicht, diese Studien detailliert zu beurteilen. - Indem ich also auf die Originalarbeit verweise, will ich mich beschränken auf ein paar kurze Bemerkungen, ferner angeben, wie ich die sogenannten Humussäuren behandele, um ihren komplizierten Charakter klarzulegen (ich verweise dabei auf meine „Bodenkundlichen Grundgedanken" S. 61 unter XXIII, wo ich die Humussäuren als einen wissenschaftlichen Lapsus bezeichnet habe) und schlieBlich nachweisen, wie man die Acidität eines kranken Bodens am einfachsten und sichersten bestimmt, um dann Abhilfe zu schaffen.

I. Bemerkungen zu den obengenannten Studien.

1. Die Forscher gehen aus vom Kasseler Braun für Studien der Humussäuren. Es läBt sich wohl kaum ein Humusmedium denken, dessen Wesen so weit von dem Pflanzennährboden abweicht, als ein Stein- oder ein Braunkohlenlager. Die Druckverhältnisse bei diesem Prozeß waren sehr hohe, sowohl durch Überlagerung als durch Gasentwicklung. Die löslichen Gärungsprodukte sind nunmehr noch im Minimum und die unlöslichen dagegen im Maximum vorhanden.

2. Es extrahieren diese Forscher mit Benzol und Alkohol im Soxhletapparat, also bei relativ niedrigerer Temperatur, um das Kasseler Braun von wachs- und harzartigen Substanzen zu reinigen. Dieser $Z$ weck kann auf diesem Wege nie erreicht werden, denn die Wachse und auch verschiedene Harze lösen sich bei gewöbnlicher Temperatur in genannten Lösungsmitteln nicht. Es bleiben also in dem gereinigten Kasseler Braun Wachse und Harze - allgemein gesprochen Phenole zurück. 
3. Die Nitrierung des gereinigten Produktes muB also auch Nitroverbindungen dieser zurückgelassenen Wachse und Harze ergeben. Zum Beweis dafür kann ich den Herren empfehlen, diese neutrale Lösung ihres rotbraunen Nitrokörpers in Natronlauge mit Natriumkarbonat zu versetzen und sie werden die Freude haben, die gelben Kriställchen von Natriumpikrat (Natriumsalz des Trinitrophenols) ausfallen zu sehen. Noch reichlicher erhält man das Produkt, wenn man das Kasseler Braun direkt nitriert, und den erhaltenen Nitrokörper wie oben angegeben behandelt. Auch das Substitutionsprodukt weist in seinem Bromgehalt keinen Widerspruch, sondern Bestätigung auf.

4. Die Herren haben uns nicht aufgeklärt über die verschiedenen quantitativen Ergebnisse ihrer Arbeit.

5. An Stücken des Kasseler Brauns hätten die Forscher das grüne Calciumellagat beobachten können. $\mathrm{Da}$ ich überhaupt nur beabsichtige, einige Anweisungen zu geben, wie die Herren Malkomesius und Albert den Erfolg hätten haben können, schon längst bekannte Sachen noch einmal am Kasseler Braun zu wiederholen, welche obendrein noch durch das Bromierungsprodukt bestätigt werden, will ich nicht näher auf diese Studien eingehen, da sie doch vollkommen außerhalb der eigentlichen Humusvorgänge liegen.

II. Zergliederung der Komplexe, welche man bisher als Humussäuren bezeichnet hat.

1. Man extrahiere das saure Material wiederholt mit siedendem Alkohol, nach der Vorschrift Fresenius' in seiner quantitativen Analyse. Aus diesem Auszuge werden die Wachse und Harze nach dieser Vorschrift ausgefällt.

a) Wachse und Harze.

Meistens gelingt es nicht, die zurückbleibende saure Lösung vollkommen klar zu bekommen, aber wohl ist sie so hell gefärbt, daß sie mit Phenolphtaleïn als Indikator mit n/10-KOH titriert werden kann.

b) Acidität

des Materials, nach Entfernung der leicht ausscheidenden Wachse und Harze. Es sei beiläufig bemerkt, dab diese Aci- 
dität für mich der Maßstab ist zur Bestimmung des erforderlichen Kalkquantums, welches man am zweckmäßigsten als Karbonat dem betreffenden Boden zusetzt.

2. Der Rest nach der Alkoholdestillation wird getrocknet und in Wasser verteilt, auf dem Wasserbade erwärmt und so lange tropfenweise $n / 10-\mathrm{KOH}$ zugesetzt, bis sich die Flüssigkeit während einer halben Stunde neutral erhält. Dieser Auszug wird mittels Zentrifugierens von dem ungelösten Rest getrennt.

Der verbleibende Rest wird mit dem äquivalenten Quantum Schwefelsäure während einer halben Stunde auf dem Wasserbad erwärmt, der erbaltene Auszug abfiltriert und der Filterrest gründlich ausgewaschen.

Die beiden Auszüge werden vereint. Diese Mischung

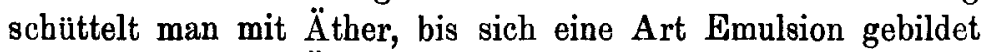
hat, d. h. bis der Äther sich nicht wieder so sclnell vom Wasser trennt. Zentrifugiert man diese Emulsion, dann werden

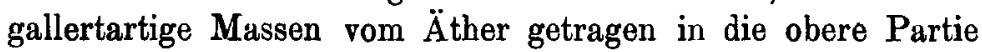
gedrängt, so daß man die mehr oder weniger dunkle Lösung mit einem Heber mit ausgezogener Spitze unter der Gallerte abführen kann. Die Gallerte verschlieBt schlieBlich selbst den Heber.

a) Die Gallerte wird vom Äther befreit, indem das Zentrifugierglas in ein erwärmtes Wasserbad gebracht wird. Sammelt man die ätherfreie Lösung und wägt man dieselbe, um sie danach einzudampfen und bei ca. $150^{\circ}$ zur Gewichtskonstanz zu trocknen, dann erhält man die Trockensubstanz, welche mit dem ausgetriebenen Wasser Kolloidmolekel bildet. Bei näherer Erforschung stellt sich die Trockensubstanz als beinahe reine Kieselsäure heraus. Wir erhalten dadurch das MaB der Wassermolekel, welche mit einer Kieselsäuremolekel als Kolloidmolekel auftreten können. Es empfiehlt sich, die ätherfreie Lösung nach Gewicht und Volum festzustellen. Durch das Trocknen erhält man den Beweis, daB mehr Gramme Wasser ausgetrieben wurden, als das Volum ccm aufwies. Mit anderen Worten: in den Kolloidmolekeln tritt das Wasser in verdichteter Form auf, welche Erscheinung uns die Benetzungswärme eines Bodens erklärt. 
c) Kolloide.

B) Die Lösung, unter der Gallerte abgezogen, wird mit einer frischen Ferrosulfatlösung tropfenweise versetzt, bis in der geklärten Lösung kein Niederschlag mehr entsteht. ${ }^{1}$ ) Der ausgefallene Niederschlag, auf dem Filter gesammelt, getrocknet, gewogen, eingeäschert und gewogen, ergibt uns das $\mathrm{MaB}$ für organische Såuren, welche in Wasser nicht lösliche Ferrosalze bilden.

d) Abkömmlinge von Gallussäure usw.

$\gamma)$ Wird die zurückbleibende klare Lösung mit n/10-KOH titriert, dann finden wir dadurch das $\mathrm{MaB}$ für die Säurenwelche sich auf dem Wasserbade aus dem Bodenmaterial ab, gespalten haben und vermutlich im Bodenmaterial (Ausgangsmaterial) nicht anwesend waren.

e) Saure Spaltungsprodukte der Operation.

Durch dieses Verfahren glaube ich deutlich darzutun, daB die sogenannten Humussäuren, welche man aus einem Alkalibodenauszuge mittels Mineralsäure ausfällt, nichts weiter sind als Absorptionskomplexe, welche sich bilden, indem $\mathrm{Kol}$ loide und Harze durch Elektrolyte zum Ausfällen gebracht werden und nun in diesem Koagulum von den verschiedensten Elektrolyten, je nach ihren spezifischen Dissoziationskonstanten, in konzentrierterem Zustande Quoten absorbieren, als sie in der wäBrigen Lösung vorkommen. (Vergl. Zeitschrift für das Forstwesen 1903, S. 736, ebenda Eebruarheft und Märzheft; wie auch in demselben Jahrgange S. 588.)

\section{Bestimmung der Acidität (Säurewert) eines}

Bodens in bezug auf das Pflanzenleben.

Indem ich hinweise auf die Arbeiten von G. Stiehr „Über das Verhalten der Wurzelhärchen gegen Lösungen“, Kiel 1903 und von Walter Maxwell „Die relative Empfindlichkeit von Pflanzen gegenüber dem Säuregehalt des Bodens", Landw. Vers. Stat. Bd 50 S. 325, muB man sein Staunen

i) Am besten bedient man sich einer bekannten Lösung des Mohr. schen Salzes. 
darüber aussprechen, wie es überhaupt möglich ist, daB man seitens der Forschung so lange Verfahren beibehalten hat, welche von der Erfahrung geradezu verurteilt werden müssen. So z. B. die Bestimmung des Säurewertes im kranken Boden, also mit träger Humusgärung. Die bis jetzt anerkannte beste Methode Fackes gibt immer noch Säurewerte, welche zu ganz enormen Ausgaben für Kalkdüngung fübren, und keineswegs biologisches Vertrauen verdienen.

Es soll hier nicht eine Kritik der Fackeschen Methode zur Bestimmung der Acidität geliefert werden, welche doch bezweckt, die erlaubte Kalkdüngung zu berechnen. Nun sollen hier mitgeteilt werden die Ergebnisse einer chemischen Arbeit, welche auf meine Veranlassung von Herrn P. Melchior unter Leitung des Herrn Dr. Coops (Dozent für Chemie an der hiesigen landwirtschaftlichen Schule) ausgeführt wurde. Herrn P. Melchior muB ich dafür hier meinen verbindlichsten Dank abstatten, während Dr. Coops die Freundlichkeit gehabt hat, die Richtigkeit der Ergebnisse, so wie ich dieselben hier mitteilen werde, zu bestätigen.

Bodenart: Trockentorf eines annähernd bundertjährigen Kiefernbestandes, welcher auf der Heide gesäet wurde; die Heide trug damals Eichenstockausschläge, welche stellenweise noch stark im Bestande vertreten sind. Die tiefergehenden Kiefernwurzeln sind durchweg erkrankt oder funktionieren nur sehr schwach, mit dem Erfolg, daß die Kiefern durchweg drehw üchsig sind.

Die organische Decke wurde am 19. Februar d. J. genommen in einem Stich von $2,25 \mathrm{dm}^{2}$.

Das Material wog $589,585 \mathrm{~g}$; nachdem es verteilt und in Flasehen untergebracht war, hatte es durch Verdunstung $6,085 \mathrm{~g}$ Wasser verloren, so daß die analytischen Ergebnisse berechnet werden müssen auf $583,500 \mathrm{~g}$ frische Substanz. Im Exsikkator über konzentrierter Schwefelsäure bei einer Dampfspannung von $20-30 \mathrm{~mm}$ getrocknet, erreichten $33,660 \mathrm{~g}$ des frisehen Materials mit 22,660 $\mathrm{g}$ Gewichtskonstanz.

Also verdunstbares Wasser $11,000 \mathrm{~g}$. - Die Einäscherung ergab 9,205 g. Daraus geht hervor:

\begin{tabular}{c|c|c|c|c}
\hline \hline & Asche & $\begin{array}{c}\text { Verdunst- } \\
\text { bares Wasser }\end{array}$ & $\begin{array}{c}\text { Organ. Sub- } \\
\text { stanz u. Struk- } \\
\text { tur-Wasser }\end{array}$ & $\begin{array}{c}\text { Aschenfreie } \\
\text { frische Sub- } \\
\text { stanz }\end{array}$ \\
\hline \hline 137,64 g fr. Substanz & 37,64 & 44,98 & 55,02 & 100
\end{tabular}


Trocknen bei $105^{\circ}$ ergab:

\begin{tabular}{c|c|c|c|c}
\hline \hline & Asche & $\begin{array}{c}\text { Trocken- } \\
\text { verlust }\end{array}$ & $\begin{array}{c}\text { Trocken- } \\
\text { substanz }\end{array}$ & $\begin{array}{c}\text { Aschefreie } \\
\text { frische } \\
\text { Substanz }\end{array}$ \\
\hline \hline $127,88 \mathrm{~g}$ fr.Substanz & 27,88 & 49,35 & 50,65 & 100
\end{tabular}

Auf diese Bodenart wurde die Methode Fackes angewandt.

$\begin{array}{lcc} & \text { I. Bestimmung } & \text { II. Bestimmung } \\ \text { Nach } 3 \text { Stunden ergaben } & 10,188 \mathrm{~g} & 10,617 \mathrm{~g} \text { frische Substanz } \\ \text { eine Entwicklung von } & 0,043 \mathrm{~g} & 0,046 \mathrm{~g} \mathrm{CO}_{2} . \\ \text { Fortsetzung ergab in } & & - \\ \text { 71 Stunden in Sa. } & 0,126 \mathrm{~g} & -\mathrm{CO}_{2} .\end{array}$

Dieses, sorgfältig übereinstimmend gehalten mit dem Material für das Trocknen im Exsikkator, kann also dementsprechend berechnet werden.

Nach diesem Ergebnis finden wir hier die sehr übereinstimmende Aciditäten auf $10 \mathrm{~g}$ frischen Materials von

$\begin{array}{ccc} & 0,0422 \mathrm{~g} & 0,0433 \mathrm{~g} \mathrm{CO} \\ \text { auf } & 3,268 \mathrm{~g} & 3,268 \mathrm{~g} \mathrm{H}_{2} \mathrm{O} \text {, }\end{array}$

durchschnittlich $0,04275 \mathrm{~g} \mathrm{CO}_{2}$, entsprechend $0,070926 \mathrm{~g}$ HCl.

Nach dieser Methode finden wir, daB das verdunstbare Wasser in Boden am 19. Februar den Säurewert von einer 2,17 prozent. Salzsäurelösung haben müBte.

In dieser Bodenart wurde der Charakter der sogenannten Humussäuren bestimmt.

$10,652 \mathrm{~g}$ frischer Boden ergaben $0,070 \mathrm{~g}$ Wachse,

$$
0,221 \mathrm{~g} \text { ausfallende Harze. }
$$

Es blieb eine Lösung zurück mit einem Säurewert von $0,4 \mathrm{ccm} \mathrm{n}$ HCl. Diese Lösung wurde mit $\mathrm{n} / 10-\mathrm{Ba}(\mathrm{OH})_{2}$ neutralisiert, wobei ein Niederschlag ausfällt. - Es verhielten sich die Baryumquantitäten, die ausgefällt werden, zu denjenigen, welche in Lösung verbleiben, wie $3: 43$.

Der trockene Rest nach der Alkoholextraktion $\quad 6,162 \mathrm{~g}$

Ausgefällte Wachse und Harze $0,291 \mathrm{~g}$

Glühverlust der trockenen Substanz in der Lösung nach Entfernung der Wachse usw.

$$
\text { Summa: } \frac{0,203 \mathrm{~g}}{6,656 \mathrm{~g}}
$$

Es wurden also vom Alkohol höchstens $10,652-6,656=3,996 \mathrm{~g}$ Wasser ausgezogen.

Dieses Muster enthielt mindestens $2,423+0,066=2,489 \mathrm{~g}$ Asche, also aschenfreie frische Sulstanz: $8,163 \mathrm{~g}$. 
Das vom Alkohol aufgenommene Wasserquantum war also mindestens $48,95 \%$ der aschenfreien frischen Substanz, also sthr nahe übereinstimmend mit dem Trocknen bei $105^{\circ}(0,4 \%$ (Differenz). - Der obige Săurewert in der Lösung nach dem Auszuge der Wachse, Harze usw. mit absolutem Alkohol, bezogen auf den Wasserverlust im Exsikkator $44,98 / 100 x / 8,163=x=3,651 \mathrm{~g}$ verdunstbares Wasser. $-0,4 \mathrm{~cm} \mathrm{n} \cdot \mathrm{HCl}=0,0144 \mathrm{~g} \mathrm{HCl}$, bezogen auf $3,651 \mathrm{~g}$ Wasser, gäbe eine Lösung von 3,95 \% Salzsäurelösung; entsprechend dem Boden. wasser, sobald die Wachse und Harze aufgeschlossen sein würden.

Der trockene Rest nach Entfernung der Wachse und Harze usw. auf dem Wasserbade blieb nach Zusatz von $8,8 \mathrm{ccm}$ n-KOH während einer halben Stunde neutral.

Diese Lösung wurde vom ungelösten Reste durch Zentrifugieren getrennt, der Rest mit gleichem Quantum n- $\mathrm{SO}_{4} \mathrm{H}_{2}$ während einer halben Stunde auf dem Wasserbad erwärmt, die erhaltene Lösung abfiltriert und mit dem alkalischen Auszuge vereint. - Es fiel kein Niederschlag aus. Die eine Hälfte wurde mit $\mathrm{K}_{2} \mathrm{SO}_{4}$ versetzt; es fiel aber nichts aus; wohl aber war die Acidität bedeutend herabgemindert, denn jetzt genügten $1,5 \mathrm{ccm} \mathrm{n} \cdot \mathrm{KOH}$ zur Neutralisierung; es müssen sich also Absorptionskomplexe gebildet haben, welche nicht direkt von der Kalilauge aufgeschlossen werden. - Die Acidität war jedoch gefallen von $8,8 \mathrm{cem} n-\mathrm{KOH}$ auf $3 \mathrm{cem}$ n-KOH.

Die andere Hälfte dieser Mischung beider Lösungen wurde mit Äther geschüttelt, bis sich der Äther nicht mehr von der Lösung abtrennte. Danach wurde abermals zentrifugiert. - Die Gallerte, welche jetzt obenauf stand, wurde mit dem Heber von der Lösung geschieden. Die Gallerte wurde ätherfrei gemacht und wog nur $8,740 \mathrm{~g}$, getrocknet $0,048 \mathrm{~g}$, und die Asche davon (beinah reines $\mathrm{SiO}_{2}$ ) wog $0,011 \mathrm{~g}$. - Es stellt sich also heraus, daB $0,011 \mathrm{~g} \mathrm{SiO}_{2}$ mit $8,729 \mathrm{~g} \mathrm{H}_{2} \mathrm{O}$ als geschlossenes Molekül auftreten kann, welche vom Äther aus der Lösung einporgebracht werden können. - Dieses Verhältnis entspricht $1 \mathrm{Mol}^{\mathrm{S}} \mathrm{SiO}_{2}$ auf 266.3 Mol. $\mathrm{H}_{2} \mathrm{O}$, so daB wir hier ein Hydrogel von $\mathrm{SiO}_{2}$ hätten mit einem Molekular-Gewicht von annähernd $48000(47990,5)$.

Die gefärbte Lösung, unter der Gallerte weggezogen, wurde in drei Teile zerlegt.

Es stellte sich heraus, daß dus Schütteln mit Äther die Acidität noch mehr herabgemindert hat, denn die drei Teile zusammen beanspruchten zur Neutralisierung im ganzen nur $1,07 \mathrm{ccm}$ n-KOH, entsprechend 2,14 ccm für die ganze Misehung, anstatt $8,8 \mathrm{ccm}$ der anfänglichen Lösung.

Ein Drittel gab mit einer Lösung des Mohrschen Salzes eine Fällung:

$$
\begin{aligned}
& 0,060 \mathrm{~g} \text { getrocknet, welche } \\
& 0,022 \mathrm{~g} \text { Asche zurücklieB. }
\end{aligned}
$$

Für die ganze Mischung hätte dies eine Fällung von $0,360 \mathrm{~g}$ Gewicht ergeben mit $0,132 \mathrm{~g}$ Asche. 
Direkte Behandlung mit $\mathrm{n} / 10-\mathrm{KOH}$ auf dem Wasserbade und Ausfällen mit einem geringen Überschuß von Schwefelsäure.

$10,716 \mathrm{~g}$ des frischen Materials blieben mit 10,6 eem n-KOH während einer halben Stunde neutral

Mit einem kleinen ÜberschuB von Schwefelsïure fiel aus der Kornplex, welchen man früher als Humussäuren bezeichnete.

Es wurde davon erbalten als Trockensubstanz $0,940 \mathrm{~g}$

mit Gesamtasche $0,042 \mathrm{~g}$.

Betrachten wir zum Vergleich die partielle Ausfällung von Wachsen und Harzen, von Kieselsäure und Stoffen unit Ferrosulfat ausgeschieden, dann ergaben 10,652 $\mathrm{g}$ der frisehen Substanz.

\begin{tabular}{|c|c|}
\hline Wachse und Harze & $0,291 \mathrm{~g}$ \\
\hline Kieselsäure & $0,288 \mathrm{~g}$ \\
\hline Fällung mit Ferrosulfat & $0,860 \mathrm{~g}$ \\
\hline $\begin{array}{r}\text { Summa : } \\
\text { : }\end{array}$ & $0,939 \mathrm{~g}$ \\
\hline
\end{tabular}

Erwägt man aber, $d a B$ in der Lösung des Alkoholextraktes, nяeh Entfernung des Alkohols, noch $0,203 \mathrm{~g}$ organischer Substanz zurückblieb, dann ist wohl kaum eine andere Annahme möglich, als daß die sogenamuten „Humussäuren" nichts weiter sind, als die Summe der Wachse, Harze, Mineralkolloïde, der Gerbstoffderivate, welche von Elektrolyten ausgefällt werden und in ibrem Koagulum umsomebr Elektrolyte absorbieren, je verdünnter die Lösung ist, aus welcher sie ausfallen. - Daher bleibt bei direktem Auszug mit Alkali und Ausfällen mit Mineralsäuren so viel weniger Asche zurück, als wenn diese Ausfällung partiell aus verschie. denen Lösungen stattfindet. Extraktion mit destilliertem Wasser und Auspressen
des Filterrestes.

26,265 $\mathrm{g}$ frisches Material enthielten, entsprechend dem Muster für das Trocknen im Exsikkator, 11,813 $\mathrm{g}$ verdunstbares Wasser.

Der Auszug batte einen Säurewert übereinstimmend mit $0,18 \mathrm{~cm}$ $\mathbf{n} \cdot \mathrm{HCl}=0,00657 \mathrm{~g} \mathrm{HCl}$. Diese Säure, auf das verdunstbare Wasser bezogen, würde einer Salzsäurelösung von $0,5561 \%$ entsprechen.

\section{Folgerungen.}

Znr Beurteilung eines Bodens mit Rücksicht auf seine Acidität, als Gefahr für die Pflanzenwurzel (speziell für die Wurzelhaare), ist ein Wasserauszug, mit Auspressen des Rẹstes in einer Schraubenpresse, neben einer Bestimmung des Wassergehaltes, das am meisten empfehlenswerte Verfahren. Zunächst ist es das natürlichste und obendrein kann jeder Praktiker solche Bestiınmung selbst ausführen. 
Zur Melioration eines kranken Bodens, wobei es besonders darauf ankommt, eine auch nur zeitweise alkalische Reaktion des Bodens zu verhüten, verfährt man am besten, indem man den Boden mit konzentriertem, heißem Alkohol auszieht. Man destilliert den Alkohol zurück und setzt Wasser hinzu, um den letzten Alkoholrest auszutreiben.

Die so erhaltene Lösung wird in zwei gleichen Teilen verteilt.

A) Die erste Hälfte wird heiß mit n/10-KOK neutralisiert. Die Operation ergebe eine Acidität von a ccm Normalsänre.

B) Aus der zweiten Hälfte läBt man die Wachse und Harze so weit wie möglich ausfallen. Man filtriert und titriert die Lösung. Diese Operation ergebe eine Acidität von b ccm Normalsäure für den Säuregrad, welcher im Boden auftreten kann, falls die Wachse und Harze aufgeschlossen werden.

Die Acidität der Harze ist in diesem Falle $(a-b)$ ccm Normalsäure.

Für das Erschlieben der Harze kann man irgend eine organische Stickstoffdüngung anwenden, sehr zweckmäBig Ahle oder auch das käufliche Ammoniumkarbonat $\left[\mathrm{NH}_{4}\right)_{2} \mathrm{CO}_{3}$ $+2\left(\mathrm{NH}_{4} \cdot \mathrm{HCO}_{3}\right]$, als Lösung angewandt. Von diesem Salz ist für jedes $\mathrm{ccm}$ Normalsäure $47,5 \mathrm{mgr}$ anzuwenden.

Die jetzt erschlossenen löslichen Säuren müssen dann aber neutralisiert werden können und zwar am zweckmäBigsten mit Calciumkarbonat. $\mathrm{Zu}$ dem Zwecke braucht man für jedes der bestimmten a ccm Normalsäure $50 \mathrm{mgr} \mathrm{CaCO}_{3}$.

Nach diesem Verfahren wird obiger Boden zu einer vergleichenden Versuchsreihe vorgerichtet. Anderenortes werden die Kulturergebnisse mit verschiedenen Sämereien von Holzarten mitgeteilt werden.

Für diese Zeitschrift glaubte ich mich auf den speziell praktisch-chemischen Teil beschränken zu müssen.

Anmerkung. Die in dieser Mitteilung veröffentlichten, analytischen Ergebnisse wurden unter Leitung des Unterzeichneten von Herrn P. Melchior im chemischen Laboratorium der hiesigen Reichlandwirtschaftlichen Schule erzielt.

Dr. G. H. Coops, Wageningen. 